The absence of reported TBE cases in professionally-menaced groups of population testifies to the efficiency of preventive services among these contingents.

\subsection{4 \\ doi: 10.15789/2220-7619-2018-4-4.24 \\ COOPERATION OF ZOOLOGICAL GROUP AND THE PCR LABORATORY FOR EVALUATION OF EPIZOOTICS IN REPUBLIC OF BASHKORTOSTAN}

A.M. Sysa ${ }^{1}$, R.R. Gazizov ${ }^{1}$, T.A. Nigmatullina ${ }^{1}$, A.A. Kazak ${ }^{1}$, A.I. Kobyakov ${ }^{1}$, O.V. Ivanova ${ }^{1}$, A.V. Moskvina ${ }^{1}$

${ }^{1}$ Center for Hygiene and Epidemiology in the Republic of Bashkortostan, Ufa, Russia

The aim of the study was to evaluate the work done to study the natural foci of tularemia in the Republic of Bashkortostan (RB).

The objectives of the study were to estimate the number of study district, the number of studies conducted, the nature of the samples studied, and the methods used.

Tularemia is a zooanthroponosis infection, characterized by the flood-marsh type of natural foci.

From 2014 to July 2018, 242 small mammals caught in the RB were examined for tularemia, of which two were infected. The first specimen was caught in the Krasnokamsky district in 2014, the second in the Gafuriysky district in 2018. These areas adjoin the natural focal point of tularemia in the city of Agidel, where in 2013, 5 cases of tularemia were reported.

Through the territory of the RB the Belaya River and its tributaries flows, therefore, in the years of active reproduction of small mammals, the dispersal of $F$. tularensis carriers along this watercourse is possible. In connection with this, the number of areas studied is also growing. In $2014-1$ district, in $2015-5$ districts, in $2016-14$ districts, in $2017-5$ districts.

Every year, the volume of conducted research, the types of investigated samples increased. Since 2016, were studied samples of water from open reservoirs, since 2017 - samples blood-sucking arthropods, and since 2018 samples of hydro fauna for research on tularemia. The total number of samples of environmental objects in 2014 was 45, in 2015 - 50, $2016-84,2017-96$, in 2018, 89 are planned.

Serologic methods (microreaction of agglutination, indirect haemagglutination reaction, inhibition of indirect haemagglutination) and PCR were used. It is planned to use the ELISA.

As the result of the study there was issued the order in the FBUZ "Center for Hygiene and Epidemiology in the Republic of Bashkortostan" about the ongoing monitoring of the epizootic condition of foci of tularemia in the area of Agidel city and the Krasnokamsky district.

4.25

doi: 10.15789/2220-7619-2018-4-4.25

\section{THE IMPACT OF GLOBAL CLIMATE CHANGE ON THE INCIDENCE OF TICK-BORNE ENCEPHALITIS IN THE EUROPEAN PART OF THE RUSSIAN ARCTIC}

N.K. Tokarevich ${ }^{1}$, A.A. Tronin ${ }^{2}$, B.R. Gnativ ${ }^{3}$, R.V. Buzinov ${ }^{4}$, O.V. Sokolova ${ }^{4}$, O.V. Blinova ${ }^{1}$

${ }^{1}$ St. Petersburg Pasteur Institute, St. Petersburg, Russia; ${ }^{2}$ Scientific Research Center for Ecological Safety, Russian Academy of Sciences, St. Petersburg, Russia; ${ }^{3}$ Center for Hygiene and Epidemiology of Komi Republic, Syktyvkar, Russia; ${ }^{4}$ Directorate of Rospotrebnadzor in Arkhangelsk Region, Arkhangelsk, Russia

The study objective was to estimate the impact of air temperature change on the incidence of tick-borne encephalitis (TBE) in the Arkhangelsk Region (AR) and in the Komi Republic (RK).

We analyzed TBE incidence rate (TBEIR) in RK in 1970-2017, and in AR in 1980-2017, its dependence both on the average annual air temperature and the local air tem- perature during the ixodid tick activity season, and satellite data on vegetation changes within the area under study.

In RK in 1970-1979, the average number of TBE cases per year was 1.4 (TBEIR was 0.1 per 100000 ), while in 2008-2017 it was 15.2 (TBEIR was 1.8 per 100000 , i.e., 18 times higher than in 1970-1979). An even sharper rise in TBEIR was registered in AR. In 1980-1989 the average number of TBE cases per year was 1.6 (TBEIR was 0.1 per 100000 ), while in 2008-2017 it was 64.4 (TBEIR was 5.4 per 100000 , i.e. 54 times higher than in 1980-1989). A sharp rise in TBEIR in the Northern Europe is due both to the significant northward shift of TBE geographical distribution limits and to TBEIR significant growth in the southern districts. During the analyzed period both average annual temperature and the air temperature during the period of tick activity increased substantially. A strong correlation was revealed between the increase in the TBEIR and the rise in the air temperature. With the help of satellite technologies a pronounced growth of the vegetation index was detected.

The increase in TBEIR within the area under study was mostly due to the air temperature increment, especially during the period of tick activity. The increase in the local vegetation index bears witness to significant changes in the entire ecosystem under the influence of climate changes that provide more favorable conditions for increase in number of animal hosts of ixodid ticks, those being the main vectors of TBE virus.

\section{PATHOGENETIC TREATMENT OF SEVERE P. FALCIPARUM MALARIA: APPROACHES TO OPTIMIZATION}

A.K. Tokmalayev ${ }^{1}$, V.B. Chentsov' ${ }^{2}$ D.V. Chernyshev ${ }^{2}$, G.M. Kozhevnikova ${ }^{1}$, N.A. Polovinkina ${ }^{1}$

${ }^{1}$ Peoples' Friendship University (RUDN University), Moscow, Russia; ${ }^{2}$ Infectious Clinical Hospital No. 2, Moscow, Russia

Pathogenesis of malaria is associated with massive destruction of erythrocytes infected with plasmodium and a development of pathological reactions. One of the most severe clinical forms of malaria is the cerebral form, which is registered in almost $10 \%$ of all P. falciparum malaria cases. This is also the leading cause of death. The aim of this study was to optimize the pathogenetic treatment of severe $P$. falciparum malaria to prevent fatal outcomes.

During the years 2007-2016 44 patients (36 men and 8 women) with severe $P$. falciparum malaria aged 16 to 69 years old were treated in the intensive care unit of Moscow state Clinical Hospital No. 2. The verification of the diagnosis was based on clinical, epidemiological history and the results of blood smears. The severity of malaria in patients was mainly due to late hospitalization: between 5 to 10 days from the onset of the disease. On admission the level of blood parasites in patients was in the range from 2500 to $2701800 \mathrm{p} / \mu \mathrm{l}$. The patients were treated in accordance with WHO recommendations $(2006,2010)$.

Ischemic damage of organs and hemorrhagic complications were prevented. In addition, a protocol of intensive care in patients with severe $P$. falciparum malaria was implemented: preventive extracorporeal hemocorrection methods were added without waiting for signs of uremia. This was carried out by a prolonged veno-venous hemodiafiltration procedure ("Prisma"), which resulted in the removal of a wide range of toxic and biologically active substances. This plasmapheresis procedure clears the plasma from fragments of dead parasites, toxic substances, and excessive amount of hemoglobin accumulated during hemolysis, thus reduces or prevents severe damage of the 
kidneys. Suppression of malaria toxicity promotes a quicker restoring of an adequate immune response of the body.

This approach of intensive care with the preventive procedure of extracorporeal hemocorrection method led to a reduction in mortality from 84 to $6.8 \%$ in patients with severe forms of $P$. falciparum malaria.

\subsection{7}

doi: 10.15789/2220-7619-2018-4-4.27

\section{CURRENT PROBLEMS IN DIAGNOSTICS AND TREATMENT OF STRONGULOIDIASIS}

A.K. Tokmalayev, N.A. Polovinkina, V.V. Konnov, K. Emerole Peoples' Friendship University (RUDN University), Moscow, Russia

Long-term observations of patients with various helminthiases showed that strongyloidiasis remains one of the most problematic regarding diagnosis of the disease. It is difficult to make a differential diagnosis on the basis of clinical symptoms due to its polymorphism.

Diagnostic errors of strongyloidiasis were discovered in 38 enrolled patients. It should be noted that incorrect diagnosis led to a change in the nosological structure of the disease. Years ago strongyloidiasis was misdiagnosed with such pathological conditions as acute or chronic enteritis, pancreatitis, bile duct dyskinesia, eosinophilic pneumonia, food poisoning, food infection, typhoid paratyphoid and nontyphoid disease. In recent years, strongyloidiasis was taken for acute leukemia, malignant tumors, Whipple's syndrome and Crohn's disease. Other investigators (N.I. Tumolskaya et al., 2014) reported about "masks" of strongyloidiasis.

Despite a comprehensive approach to laboratory diagnostics of chronic strongyloidiasis according to guidelines, examination of stool specimens (baermann technique) and investigation of the duodenal contents are rarely implemented in clinical laboratories. Therefore parasitological diagnosis of helminthiasis often is established with a significant delay.

Ivermectin is currently the drug of choice in the treatment of strongyloidiasis. It is suitable for the treatment of acute, chronic and disseminated forms of the diseases. A Nobel Prize in 2015 was awarded for its discovery. In the Russian Federation, ivermectin is neither registered nor produced. An alternative drug albendazole is used with a daily dose of 400-800 mg 1-2 times for 3 days. Albendazol is a drug of foreign origin and is available all over the country. Its effectiveness is insufficient and in some cases repeated courses of treatment are required. With early diagnostics and treatment with effective anthelmintic drugs and adequate rehabilitation pathogenetic therapy, the prognosis is usually good, with the exception of immune compromised cases (HIV/AIDS, tuberculosis, non-specific inflammatory diseases, etc.).

\section{ANALYSIS OF POPULATIONS OF BACILLUS ANTRACIS} STRAINS ON THE BASIS OF THEIR RESISTANCE TO SPECIFIC ANTHRAX BACTERIOPHAGES

\section{O.I. Tsygankova, E.A. Koteneva, A.V. Kalinin}

Stavropol Plague Control Research Institute, Stavropol, Russia

The specific anthrax bacteriophage lysis is a compulsory test in the scheme of identification of $B$. anthracis strains, however it does not enable us to estimate quantitatively the presence of phage resistant clones in the population of the strain.

The aim of the study was to investigate the population composition of two virulent strains of $B$. anthracis on the base of phage resistance to specific bacteriophages.
Spore suspensions of typical virulent $B$. anthracis strains 1 (SO) and 81/1 were used as suspensions in a $30 \%$ glycerin solution kept in sealed ampoules at $4-6^{\circ} \mathrm{C}$ for more than 20 years. Concentrations of phage corpuscles in experimental batches of bacteriophages Gamma A-26, BA-9, K-VIEV were, correspondingly, $8 \times 10^{9}, 4 \times 10^{8}$ and $2 \times 10^{8}$ per $1 \mathrm{ml}$. Accurately $0.1 \mathrm{ml}$ of a spore suspension in a concentration of $1 \times 10^{3}$ were applied to Hottinger's agar and spread over its surface. When the liquid was absorbed completely, one of the bacteriophage preparations was applied to test plates, moistening the whole surface of plates. Plates which were not treated with bacteriophage preparations served as controls.

Not a single colony grew on plates of both strains treated with bacteriophage Gamma A-26. Plates treated with bacteriophage K-VIEV showed a $2.9 \%$ growth of colonies of the control of the strain B. anthracis 1 (SO) and a $4.8 \%$ growth of colonies of the strain B. anthracis 81/1. Plates treated with phage BA-9 showed a $10.9 \%$ growth of colonies of the strain $B$. anthracis $1(\mathrm{SO})$ and a $17.3 \%$ growth of colonies of the strain B. anthracis 81/1, correspondingly. For further determination of sensitivity to all the three bacteriophages we used 12 colonies of each strain, which showed resistance to phages K-VIEV and BA-9 at the first stage.

The retest showed that in both strains $16.7 \%$ of variants separated on the base of their resistance to bacteriophage BA-9 were sensitive to all the three bacteriophages. Among variants of the strain $B$. anthracis $81 / 1$ which were selected from the plates treated with bacteriophage BA-9 such variants made up $20 \%$, and in B. anthracis 1 (SO) - 10\%. Among variants of both strains variants resistant to the action of bacteriophages Gamma A-26 and BA-9 and sensitive to bacteriophage K-VIEV were found. Among variants of $B$. anthracis 1 (SO) selected from cultures treated with bacteriophage BA-9, $80 \%$ were sensitive to bacteriophages BA- 9 and Gamma A-26 and resistant to bacteriophage K-VIEV.

4.29 doi: 10.15789/2220-7619-2018-4-4.29

\section{COMPARATIVE CHARACTERIZATION OF SUBCULTURES ISOLATED FROM A POPULATION OF BACILLUS ANTHRACIS 1 (SO) STRAIN ON THE BASIS OF PHAGE RESISTANCE TO SOME SPECIFIC ANTHRAX BACTERIOPHAGES}

O.I. Tsygankova, E.A. Koteneva, A.V. Kalinin

Stavropol Plague Control Research Institute, Stavropol, Russia

On the basis of many phenotypic properties the causative agent of the anthrax shows not only intraspecific variability between strains, but also intrapopulation variability in some strains.

The aim of the work was to study a complex of phenotypic properties and genetic characteristics of variants of the virulent strain $B$. anthracis 1 (SO) in the group of isolated on the basis of resistance to specific anthrax bacteriophages Gamma A-26, BA-9, K-VIEV and to carry out their comparative analysis.

We used the initial strain B. anthracis 1 (SO). Concentrations of phage corpuscles in experimental batches of bacteriophages Gamma A-26, BA-9, K-VIEV were, correspondingly, $8 \times 10^{9}, 4 \times 10^{8}$ and $2 \times 10^{8}$ per $1 \mathrm{ml}$. The criterion for selection of cultural variants was their resistance to bacteriophages. Phenotypic properties and genetic characteristics of isolated subcultures were defined according to the Guidelines 4.2.2413-08.

After treatment of spore cultures on Hottinger's agar with each of the bacteriophages separately, incubation for 24 hours at $37^{\circ} \mathrm{C}$, phage resistant cultural variants being distinguished from the initial typical strain by capsule formation and toxin production, hemolytic, proteolytic 